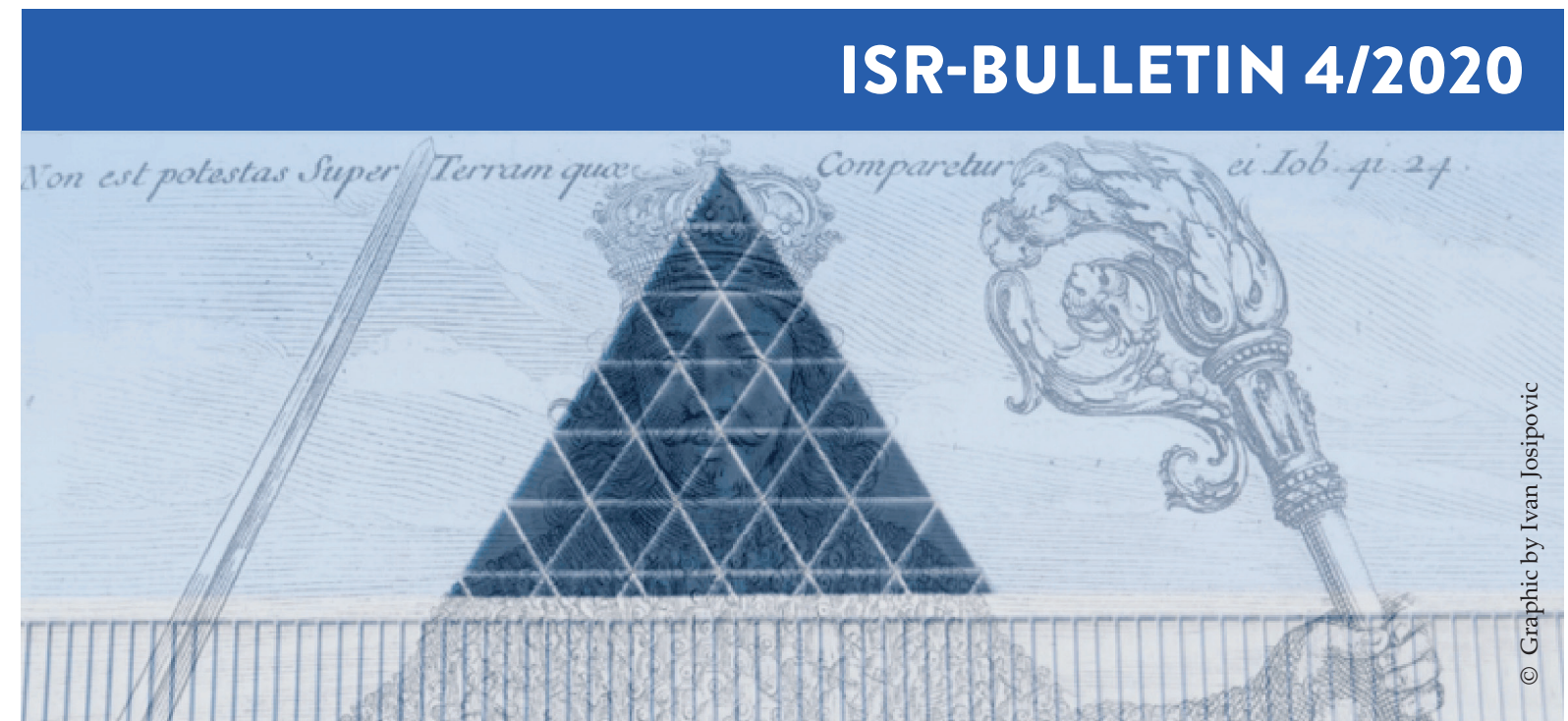

\title{
WARUM DER SOMMER DER MIGRATION 2015 IN ERSTER LINIE EINE POLITISCHE KRISE AUSLÖSTE
}

\section{IVAN JOSIPOVIC \& URSULA REEGER (ISR)}

Das Forschungsprojekt RESPOND untersucht - neben vielen anderen Themen - den politischen Umgang mit Fluchtzuwanderung nach Österreich, insbesondere im Zusammenhang mit dem Krisenjahr 2015. Gemeinsam mit 13 anderen Projektpartnern analysieren Ivan Josipovic und Ursula Reeger europäische und nationalstaatliche Asyl- und Integrationspolitiken der vergangenen Jahre. Dazu wird ein interdisziplinärer Ansatz gewählt, der sich neben der Analyse gesetzlicher Reformen und politischer Debatten auch dafür interessiert, was bestimmte rechtliche und politische Rahmenbedingungen für direkt und indirekt betroffene Personengruppen bedeuten. Dies sind zum einen Geflüchtete selbst und zum anderen ExpertInnen, die beruflich im Bereich Asyl und Integration tätig sind. Im ersten Schritt der Forschung ging es darum, die politischen Dimensionen der vielfach konstatierten Krise von 2015 abzustecken.

\footnotetext{
KEYPOINTS • Die Wahrnehmung einer Krise im Kontext der Fluchtzuwanderung von 2015 ist mitunter in Konflikten innerhalb des politischen Systems und dessen Problembearbeitung zu verorten.

- Der Sommer der Migration 2015 hat Parteien in deren inhaltlicher und personeller Ausrichtung stark beeinflusst und somit inner- und zwischenparteiliche Konflikte losgetreten.

- Ebenso traten Konflikte zwischen Regierungsebenen hervor, etwa zwischen der EU- Kommission und der österreichischen Bundesregierung, wenn es um Schengen geht, oder zwischen Bund und Ländern, wenn dies die innerstaatliche Verteilung von Geflüchteten betrifft.

- Liberale Demokratien sind gerade in grundrechtssensiblen Bereichen wie der Asylpolitik rechtsstaatlichen Zwängen unterworfen. Restriktive Maßnahmen führen somit häufig zu komplexen und fragmentierten gesetzlichen Rahmenwerken und sind anfällig für Prüfungen durch Höchstgerichte.
} 


\section{WIE KAM ES ZUR ÖFFENTLICHEN WAHRNEHMUNG EINER KRISE?}

Zwischen 2015 und 2016 nahm Österreich eine beachtliche Anzahl von Asylsuchenden auf, vorwiegend aus dem Nahen Osten und aus Afghanistan. Alleine im genannten Jahr wurden mehr als 88.000 Anträge auf Asyl gestellt. Diese Zuwanderungswelle beeinflusste den politischen Alltag nachhaltig, und das trotz einer historisch gewachsenen Tradition als Einwanderungsland. Doch was konstituiert eine öffentliche Wahrnehmung von „Krise“, also eine Situation der Diskontinuität, in der gesellschaftliche und politische Konflikte über bestehende institutionelle Strukturen und Steuerungsmaßnahmen entbrennen? Im Rahmen des Projekts RESPOND argumentieren wir, dass die Krise nicht alleine der Anzahl der Asylsuchenden geschuldet ist, sondern dass sie sich vornehmlich als Konflikt innerhalb der politischen Problembearbeitung von Asylfragen ergibt.

\section{RAPIDER ANSTIEG VON ASYLANTRÄGEN}

Freilich war das Krisenmoment im engeren Sinn eine transnationale humanitäre Krise, die sich in Österreich in einem rapiden Anstieg von Asylanträgen manifestierte und kurzfristig Probleme in der Unterbringung verursachte. Die Zahl der Anträge kletterte von 14.416 Anträgen im Jahr 2011, auf 28.064 im Jahr 2014 und erreichte einen Höchststand im Jahr 2015 als rund 88.340 Personen einen Asylantrag stellten.

\section{JENSEITS BLOSSER ZAHLEN: ZUWANDERUNG BEEINFLUSST DIE POLITIK}

Ab der Mitte des Jahres 2015 erreichten Menschen in großen Gruppen entweder zu Fuß oder mit Zügen und Bussen über Ungarn und Slowenien das österreichische Bundesgebiet. Das öffentlich vermittelte und mediatisierte Bild von Geflüchteten stand dabei in einem scharfen Kontrast zu vorherrschenden Vorstellungen von Passivität und Vulnerabilität von Personen, die sich sonst tendenziell unsichtbar über Grenzen hinwegbewegen. Diese Ausnahmesituation war nun mit einem langfristigen parteipolitischen Trend erhöhter politischer Aufmerksamkeit für bestimmte grenzüberschreitende Phänomene gekoppelt. Dies wird auch als Globalisierungskonfliktlinie in der Parteienforschung bezeichnet, nämlich dauerhaften politischen Konflikten über soziale, politische und wirtschaftliche Prozesse, die nationalstaatliche Grenzen erodieren. Zuwanderung über das Asylsystem bedient eine solche Konfliktlinie auf zweierlei Weisen: Hier fällt die Wahrnehmung eines Kontrollverlustes über staatliche Grenzen mit einer vollzogenen Abgabe von Souveränität an inter- und supranationale Entitäten wie die EU zusammen. Die Entwicklungen ab 2015, als Menschen auf der Suche nach Asyl und einem sichereren Leben entgegen den europäischen Dublin-Bestimmungen auf sichtbare Weise Grenzen überschritten, führten zu einer starken Politisierung des Themas und initiierten einen Wandel innerhalb politischer Strukturen und wichtiger kollektiver Akteure wie der politischen Parteien.

\section{AUFGRIFF DES THEMAS FLUCHT DURCH POLITISCHE PARTEIEN}

Das Jahr 2015 war eine sogenannte „critical juncture“ für politische Parteien in Österreich, also eine entscheidende Situation der Unsicherheit, die wegweisend war für die folgende inhaltliche und personelle Ausrichtung der Parteien. Dies war besonders im Fall der Österreichischen Volksparteien sichtbar. In der programmatischen Profilierung der Partei verschob sich der traditionelle Vorrang wirtschaftspolitischer Belange unter dem aktuellen Bundeskanzler Sebastian Kurz zu Gunsten von dessen politischer Vision mit dem Themenschwerpunkt "Ordnung und Sicherheit" im Bereich der Zuwanderung. Vorangegangene Studien zeigen, wie die ÖVP einen Großteil der Positionen der Freiheitlichen Partei in Bezug auf Einwanderung und Integration übernahm. Das Thema Asyl sorgte sowohl innerhalb des damaligen Koalitionspartners SPÖ also auch zwischen SPÖ und ÖVP für neues Konfliktpotential. Die vorgezogene Nationalratswahl 2017 entwickelte sich folglich zu einem Deutungskampf über migrationspolitische Entwicklungen seit 2015. Als Gewinner gingen konservative und rechte Kräfte hervor. Die daraus resultierende Umgestaltung der Bundesregierung unter ÖVP und FPÖ mündete in einen neuen Zyklus der Einwanderungs- und Integrationspolitik.

\section{KONFLIKTE ZWISCHEN REGIERUNGSEBENEN}

Das Thema Asyl sorgte nicht nur innerhalb und zwischen politischen Parteien für Konfliktpotential, sondern auch zwischen verschiedenen Regierungsebenen. Der erste Konflikt spielte sich zwischen der EU und Österreich ab. Im September 2015 führte Österreich wieder systematische Grenzkontrollen an den Grenzübergängen zu Slowenien und Ungarn ein und forderte damit ein Stück nationalstaatlicher Souveränität zurück. Diese Kontrollen wurden fortwährend verlängert, während die EU-Kommission angesichts fallender Asylantragszahlen wiederholt zur Rückkehr zum normalen Schengen-System mahnt. Ebenso bekannte sich Österreich zunächst zum EU-Relocation-Programm, jedoch wurde von den ursprünglich vereinbarten 1.953 Personen nur ein geringer Bruchteil aus Italien und Griechenland aufgenommen.

Der zweite Konflikt betriff die Aufnahme von Asylwerber/ Innen innerhalb Österreichs. Diese wurde seit 2004 zwischen dem Bund und den Ländern über die Grundversorgungsvereinbarung geregelt. Demnach verpflichten sich die Länder, AsylwerberInnen nach einer von der Bevölke- 
rungszahl abhängigen Quote aufzunehmen. Im Jahr 2015 waren einige Bundesländer und Gemeinden nicht bereit, Wohnraum zur Verfügung zu stellen, oder sie stießen auf Widerstand in der Öffentlichkeit. Daraus entwickelte sich ein offener Konflikt mit der Bundesregierung, der zur Verabschiedung eines Verfassungsgesetzes führte. Das sogenannte Durchgriffsrecht erlaubte dem Bund, eigene Unterkünfte in bundeseigenen Immobilien zu betreiben.
Anspruch elektoraler Demokratien ergibt, die Präferenzen der Mehrheitsbevölkerung politisch zu artikulieren. Während die Gewährung von Asyl also unter dem Elektorat aus diversen Gründen auf mehr oder weniger Zustimmung stoßen mag, ist das Regierungshandeln liberal-demokratischer Staaten qua Selbstdefinition durch bestimmte universelle Grundrechte und durch Verpflichtungen auf internationaler Ebene eingeschränkt. Restriktive Maßnahmen führen somit häufig zu komplexen und fragmentierten gesetzlichen Rahmenwerken und sind anfällig für Prüfungen durch Höchstgerichte. Dies zeigte sich etwa im Zusammenhang mit Restriktionen für Beschwerdefristen gegen Asylentscheidungen oder mit der Konditionalisierung von Sozialhilfeansprüchen von Asylberechtigten.
Graffito in einer Unterführung nahe beim Hauptbahnhof Wien

(c) Foto von Ursula Reeger

\section{LIMITIERTE PROBLEMBEARBEITUNG: POLITIK BEEINFLUSST ZUGEWANDERTE}

\section{DIE BESCHRÄNKUNGEN NATIONALSTAATLICHEN HANDELNS}

Die erhöhte Fluchtzuwanderung des Jahres 2015 führte nicht nur zu Krisenerscheinungen bei politischen Akteuren und in etablierten Strukturen. Ebenso erwies sich die politische Gestaltung künftiger Zuwanderung und Integration als durch tiefe Widersprüche geprägt. Besonders in einem grundrechtssensiblen Bereich wie Asyl ist (national-)staatliches Handeln einigen wichtigen Beschränkungen unterworfen, sofern es sich an liberal-demokratische Grundsätze hält. Liberale Demokratien sind nämlich zwischen zwei gegensätzlichen Grundüberlegungen hin- und hergerissen. Zum einem existiert ein rechtsbasierter Grundgedanke, der weitgehend auf verfassungsmäßig gewährleisteten Grundrechten, internationalen Verträgen sowie dem Primär- und Sekundärrecht der EU fußt. Auf der anderen Seite besteht ein mehrheitsbasierter Grundgedanke, der sich aus dem

\section{AUSBLICK: WAS BEDEUTEN DIVERSE STEUERUNGSMASSNAHMEN FÜR DIE BETROFFENEN?}

RESPOND widmet sich neben der Analyse gesetzlicher Reformen und politischer Debatten auch der Frage, was bestimmte rechtliche und politische Rahmenbedingungen für direkt und indirekt betroffene Personengruppen bedeuten. In diesem Sinn blicken wir auf die Implementierungsebene von Steuerungsmaßnahmen in den Bereichen Grenzmanagement, Flüchtlingsgrundversorgung, Asylverfahren und Flüchtlingsschutz sowie Integration. Mittlerweile hat das RESPOND-Team insgesamt fünf Länderberichte zu den genannten Themen verfasst. Darin geht es um die Wahrnehmungen von Geflüchtete selbst als auch von ExpertInnen, die professionell im Bereich Asyl und Integration tätig sind.

Konsens bestand bei WissenschaftlerInnen, PraktikerInnen und Geflüchteten darüber, dass es sinnvoll ist, bei allen Geflüchteten Integrationsbemühungen so früh wie möglich zu setzen, also bei AsylwerberInnen bereits während des laufenden Asylverfahrens, wenngleich zu diesem Zeitpunkt noch nicht entschieden ist, ob der betreffenden Person dauerhaft Aufenthalt in Österreich zuerkannt werden wird. 


\begin{tabular}{|c|c|}
\hline ECKDATEN & $\begin{array}{l}\text { Projektteam: Ivan Josipovic, Ursula } \\
\text { Reeger (ISR) } \\
\text { Auftraggeber: Europäische Union, } \\
\text { H2020, GA } 770564\end{array}$ \\
\hline $\begin{array}{l}\text { ZUM } \\
\text { WEITERLESEN }\end{array}$ & $\begin{array}{l}\text { Josipovic, I., Reeger, U. and Rottman, S } \\
\text { (2020): Beyond Legal Status: Exploring } \\
\text { Dimensions of Belonging among Forced } \\
\text { Migrants in Istanbul and Vienna, Social } \\
\text { Inclusion, Vol.8, No.1. } \\
\text { Josipovic, I. and Reeger, U. (2019): Die } \\
\text { Auswirkungen der „Flüchtlingskrise“ } \\
\text { des Jahres } 2015 \text { in Österreich: Politische } \\
\text { Reaktionen und Einschätzungen von Ex- } \\
\text { pertInnen aus der Praxis. In: R. Bauböck, } \\
\text { C. Reinprecht und W. Sievers (Hg.). } \\
\text { Flucht und Asyl - Internationale und } \\
\text { österreichische Perspektiven. Online: } \\
\text { https://austriaca.at/8496-6inhalt?fra- } \\
\text { mes=yes. } \\
\text { Josipovic, I. and Reeger, U.(2018, 2019, } \\
\text { 2020): Country Reports Austria. Global } \\
\text { Migration: Consequences and Respon- } \\
\text { ses - RESPOND Working Paper Series. } \\
\text { Vienna. }\end{array}$ \\
\hline KONTAKT & $\begin{array}{l}\text { Ivan Josipovic, MA } \\
\text { T: }+431 \text { 51581-3524 } \\
\text { ivan.josipovic(at)oeaw.ac.at }\end{array}$ \\
\hline
\end{tabular}

Impressum

Medieninhaber: Österreichische Akademie der Wissenschaften, Dr. Ignaz Seipel-Platz 2, 1010 Wien. Herausgeber: Institut für Stadt- und Regionalforschung, Postgasse 7/4/2, 1010 Wien I Erscheinungsweise: halbjährlich | Satz: Mag. Florian Partl | Grafik, Design: ÖAW, ISR Verwendbarkeit/Copyright: ISR | ISSN: 2708-9282 